\title{
THE INTERNET OF THINGS: AN OVERVIEW OF SELECTED SMART HOME TECHNOLOGY
}

\author{
Calandra E. Weaver, Ball State University, ceweaver@bsu.edu \\ Edward J. Lazaros, Ball State University, ejlazaros@bsu.edu \\ Jensen J. Zhao, Ball State University, jzhao@bsu.edu \\ Christopher B. Davison, Ball State University, cbdavison@bsu.edu \\ Allen D. Truell, Ball State University, atruell@bsu.edu
}

\begin{abstract}
The purpose of this paper is to provide an overview of selected smart home Internet of Things (IoT) technologies and their uses. Specifically, the smart home technology uses section provides (1) an explanation of smart home technology, (2) a connection to the IoT, and (3) an overview of various types of devices. Then, the section on advantages and disadvantages highlights some of the technology's conveniences and purposes as well as its risks. This section details the privacy risks of voice assistant devices. Lastly, the section on installation overview of selected devices provides technical insights, such as price and installation information, about voice assistants, Ring Doorbells, and smart lights.
\end{abstract}

Keywords: Smart home technology, Internet of Things (IoT), and Technology Users

\section{INTRODUCTION}

Houses with voice control and automated features are not futuristic anymore as homeowners are implementing smart home technologies in their residences. These devices serve various purposes and offer various features depending on the type of device and the brand. Potential users may want to better understand the basis of these devices before purchasing, so an overview of voice assistant devices, Ring Doorbells, and smart lights is provided, specifically detailing their uses, advantages, disadvantages, and installation process.

\section{SMART HOME TECHNOLOGY USES}

According to Furchgott (2016, p. 1) from The New York Times, smart home technology is any household appliance or device with a connection to the Internet. This technology utilizes the "Internet of things" (IoT), which is the concept of connecting the Internet to any type of device with an on/off feature (Morgan, 2014). Smart home technology uses automation to modify features in a home, which can solve problems or inconveniences (Colon \& Griffith, 2019; Cooper, 2019; Furchgott, 2016, p. 1). Some examples of smart home technology include voice assistant devices, Ring Doorbells, and smart lights.

Voice assistant devices are a type of smart home technology that receives commands from their users (Wojciechowski, 2019, p. 33). Examples of voice assistant devices include Amazon Alexa, Google Assistant, and Siri. On mobile devices, voice assistants include Siri, Google Now, and Cortana (Pullen, 2015). Voice assistant devices utilize the IoT in the form of a speaker or a mobile device and contain computer chips with microphones for users to give verbal commands or ask questions to the device (Wojciechowski, 2019, p. 33, 35). Wi-Fi connects the commands or questions to a computer, which then transfers them to "the cloud." Then, the device responds to the command or question typically in a soft, female voice (Dennon, 2019; Wojciechowski, 2019, p. 33, 35).

Users connect their Ring Doorbell to their Wi-Fi at home and use the Ring app to view the Ring Doorbell's live video footage, which is saved in the cloud (Derrick, 2019). Additionally, users receive alerts when it detects motion from within 30 feet outside the door, and they can talk to whomever is at their door even while they are away from home (Derrick, 2019; Watters \& House, n.d.). Users can connect their Ring Doorbell to Amazon Alexa devices (Ring, 2020b).

Smart lights connect to the power at a user's home, but they also connect to an app allowing users to control them at any time and automate them (Philips Hue, 2019). Smart lights can also connect to a voice assistant enabling users to control their lighting with their voice. Smart lights can be controlled individually or as a group, and users can save different light settings for different activities or situations, such as eating dinner or reading a book (Marshall, 2017; 
Philips Hue, 2019). Users may have the option to dim their lights or adjust them from warm to cool or from white to color (Philips Hue, 2019).

\section{ADVANTAGES AND DISADVANTAGES}

Most current research about smart home technology focuses on areas including engineering, design, and health care, but little focus is placed on its users (Wilson et al., 2014, p. 473). Wilson et al. (2017, p. 79) identified that users can use smart home technology for security, energy-saving, and other practical purposes, but they can also use it for entertainment purposes. For instance, Amazon Alexa devices respond to an array of commands including practical commands such as calling and messaging and entertainment commands such as playing music or adjusting music volume (Priest et al., 2020). Ring Doorbells are used for home security and decreasing neighborhood crimes (Ring, 2020a). Smart lights save energy because they produce less heat and work longer than traditional light bulbs (Philips Hue, 2019).

In the past, early adopters were the primary users of smart technology, but it is becoming increasingly mainstream (Welinder, 2019). In 2018, 41 percent of U.S. homes had Alexa or Google Assistant, and this is double what it has been in the past. The competition between Amazon, Google, and Apple is one factor contributing to the increase in usage. Also, popular hardware companies are experimenting with and implementing smart home technology, which shows that smart home technology is becoming trendier and increasing in demand. According to Nikou (2018, p. 16), people are more likely to use smart home technology if they are aware of other people who are using it. Potential users are also attracted to the "newness" and "innovativeness" of the technology (Nikou, 2018, p. 16). However, high prices can turn potential users away from purchasing smart home technology. User's daily schedules may affect whether they benefit from the technology or not because in Oliveira et al.'s (2019, p. 11) study of smart thermostats users, participants with more flexible schedules and active home found the device to be more useful than participants with more structured schedules and less active homes.

Moreover, users question how smart home technology will affect their privacy because they give up some privacy and control with smart home technology's cameras, microphones, and sensors (Wilson et al., 2014, p. 473-474). For example, voice assistants have caused privacy issues for some users. Voice assistant devices listen for users to say a "wake word" to wake up the device before saying a command (Alepis \& Patsakis, 2017, p. 17,841; Ford \& Palmer, 2018, p. 68). Ford and Palmer (2018, p. 78) found that Amazon Echos can listen to and record conversations even when users have not spoken the wake word. Echos sometimes mistake a word for the wake word and begin listening to a conversation. Users can try to prevent this through voice recognition training, so their voice assistant device can better recognize their voice. Users have to approve of various permissions allowing voice assistants to access certain features, but sometimes voice assistants do not ask for permission, which is a potentially dangerous invasion of privacy (Alepis \& Patsakis, 2017, p. 17,841). For instance, Echos have ordered products online because they misheard someone talking on TVs (Pfeifle, 2018, p. 422). Echos have also recorded conversations about criminal activity. Amazon can release recorded conversations to the police because the conversations are not protected by the 4th Amendment (Ford \& Palmer, 2018, p. 78). Users can prevent recordings by manually switching their voice assistants off, but then, users will need to manually turn on their voice assistants when they want to use it again. If users manually turn their devices on and off, they may feel they are not using their device to its full potential because users usually like activating their voice assistants through voice alone.

\section{INSTALLATION OVERVIEW OF SELECTED DEVICES}

Amazon Alexa voice assistant is utilized in the Amazon Echo, a smart speaker (Greenwald, 2019). As of January 2020, the third-generation, and latest generation, Amazon Echo is listed as $\$ 99.99$ (Amazon, 2020). To set up, users first need to download the Amazon Alexa app on a smartphone, tablet, or other mobile device before plugging the Echo into an electrical socket (Amazon, 2019). Then, using the Alexa app, users can add their Echo to the app and begin connecting it to their Wi-Fi (Porter, 2019). With the app, users can add calling and messaging and set up other features, such as their lists, music, news, or settings (Amazon, 2019). Once the Echo is connected to the Internet, Alexa can answer questions and perform tasks, such as providing reminders (Dennon, 2019). Alexa is learning more efficiently than other voice assistants. She has a Follow-up Mode for users to ask multiple questions in a row. Because Alexa has a greater range of response prompts, she can respond inaccurately. Dennon (2019) rated Alexa as the best 
voice assistant device for device compatibility, meaning that she can connect with other smart home devices. Alexa exceeds its competition because approximately 7,400 brands with smart home devices are compatible with Alexa while Google Assistant is compatible with about 1,000 brands, and Siri is compatible with about 50. On the downside, whereas Google Assistant and Siri are preinstalled on smartphones, Alexa is not. Users can use voice assistance through the Amazon Alexa app, but Google Assistant and Siri users can skip this step.

Ring Doorbell offers various versions of their video doorbell. As of January 2020, the traditional video doorbell is list as \$99.99, and the other versions have higher prices: the video doorbell 2 for $\$ 199$, the video doorbell pro for \$249, and the video doorbell elite for \$499 (Ring, 2020c; Ring, 2020d; Ring, 2020e; Ring, 2020f). Before installing the Ring Doorbell, homeowners need to download the Ring app, available for Android, iOS, and Windows devices, to create an account and connect their device to the app (Derrick, 2019; Ring, 2020g). If users already have a doorbell, they can remove their doorbell and attach the mounting bracket, which comes with their Ring Doorbell, in the previous doorbell's place (Ring, 2020h). Then, homeowners should take the wires from the previous doorbell and wrap them around the screws from the mounting bracket. Finally, the Ring Doorbell fits over the mounting bracket, and when the Ring Doorbell is pressed, a sound will chime inside their house. If users do not already have a doorbell, they can attach the mounting bracket directly to the wall next to their door, and then the Ring Doorbell fits over the mounting bracket (Ring, 2020i). The Ring Doorbell sends notifications to their phone about activity at their door, but because previous doorbell wires do not connect to this Ring Doorbell, it does not chime inside their home (Ring, 2020j). Users can purchase the Ring Chime, which plugs into power outlets and will create a chime sound when the Ring Doorbell is pressed (Ring, 2020k).

Philips Hue is the most popular brand of smart lighting, but other brands include IKEA and Hive (Marshall, 2017). For first-time users, Philips Hue offers a starter kit. As of January 2020, the white starter kits included four warm white lights, a bridge, an Ethernet cable, and a power adapter and is listed as $\$ 99.99$, and they listed an individual warm white light bulb as $\$ 9.99$ (Lloyd, 2017; Philips Hue, 2020a; Philips Hue, 2020b). To set up a starter kit, users need to screw their Philips Hue light bulb and turn on the lights from the light fixture or switch (Lloyd, 2017). Then, they must connect the power adapter into the bridge and an outlet before connecting the Ethernet cable into the bridge and their router. Users should download the Philips Hue app, which should connect to their bridge, and they can begin setting up their lights. If users want to adjust their lights' warmness and coolness or color, they must additionally purchase white ambiance or white and color ambiance bulbs, and users need to purchase a dimmer switch to adjust dimness (Philips Hue, 2020c; Philips Hue, 2020d; Philips Hue 2020e).

\section{SUMMARY}

This paper intended to overview smart home IoT technologies, so readers should have a better understanding of its types and uses. In summary, smart homes technology enhances a home, providing convenience and entertainment. Users can customize their homes by adjusting, automating, and programing their setting preferences, but users should be aware of the technology's potential privacy risks. This paper also uses the Amazon Echo, Ring Doorbell, and Philips Hue lights as examples to illustrate the uses, benefits, and installation process of specific devices.

\section{REFERENCES}

Alepis, E., \& Patsakis, C. (2017, September 27). Monkey says, monkey does: Security and privacy on voice assistants. IEEE Access, 5, 17, 841-17,851. https://doi.org/10.1109/ACCESS.2017.2747626

Amazon. (2019). Amazon Echo (3 ${ }^{\text {rd }}$ Generation): Quick start guide. [PDF]. Amazon. https://customerdocumentation.s3-us-west-

2.amazonaws.com/Alexa+Devices/Echo_Gen3_Online_QSG_ENG.pdf

Amazon. (2020, January). All-new Echo ( $3^{r d}$ gen) - smart speakers with Alexa - sandstone. Amazon. https://www.amazon.com/all-new-Echo/dp/B07PBGN2WX

Colon, A., \& Griffith, E. (2019, November 19). The best smart home devices for 2019. PCMag. https://www.pcmag.com/article/303814/the-best-smart-home-devices-for-2019 
Cooper, A. (2019, March 22). The emerging trend of smart home technology in real estate. Entrepreneur. https://www.entrepreneur.com/article/331001

Dennon, A. (2019, July 10). The best voice assistants. Reviews.com. https://www.reviews.com/voice-assistant/

Derrick, A. (2019, December 13). What is the ring doorbell and how does it work? Lifewire. https://www.lifewire.com/how-ring-doorbell-works-4583925

Ford, M., \& Palmer, W. (2018, June 28). Alexa, are you listening to me? An analysis of Alexa voice service network traffic. Personal and Ubiquitous Computing, 23(1), 67-79. https://doi.org/10.1007/s00779-018$1174-\mathrm{x}$

Furchgott, R. (2016, July). Smart homes, now simpler: Technology improvements make home automation more accessible and affordable. New York Times, pp. 1, 9.

Greenwald, W. (2019, October 18). Amazon Echo (3 ${ }^{\text {rd }}$ generation). PCMag. https://www.pcmag.com/review/371360/amazon-echo-3rd-generation

Lloyd, C. (2017, July 5). How to set up your Philips Hue lights. How-To Geek. https://www.howtogeek.com/247500/how-to-set-up-your-philips-hue-lights/

Marshall, C. (2017, July 9). What is smart lighting? Everything you need to know for your connected home. TechRadar. https://www.techradar.com/news/what-is-smart-lighting-everything-you-need-to-know-foryour-connected-home

Morgan, J. (2014, May 13). A simple explanation of 'the Internet of things'. Forbes. https://www.forbes.com/sites/jacobmorgan/2014/05/13/simple-explanation-internet-things-that-anyonecan-understand/\#5053d17b1d09

Nikou, S. (2018, June). Internet of Things: Exploring households' intention to use smart home technology. Telecommunications Policy, 1-23. Retrieved July 2, 2020.

Oliveira, L., Mitchell, V., \& Andrew, M. (2019, August). Smart home technology—comparing householder expectations at the point of installation with experiences 1 year later. Personal and Ubiquitous Computing, 1-14. https://doi.org/10.1007/s00779-019-01302-4

Pfeifle, A. (2018, March). Alexa, would should we do about privacy? Protecting privacy for users of voice activated devices. Washington Law Review, 93(1), 421-458. Retrieved July 1, 2020.

Priest, D., Dyson, T., \& Martin, T. (2020, February 21). Every Alexa command to give your Amazon Echo smart speaker or display. CNET. https://www.cnet.com/how-to/every-alexa-command-to-give-your-amazonecho-smart-speaker-or-display/

Porter, J. (2019, October 17). How to set up your new Amazon Echo. TechRadar. https://www.techradar.com/how-to/amazon-echo-setup

Pullen, J. P. (2015, October 1). 10 ways your phone's voice assistant can make you more productive. Time. https://time.com/4057993/siri-google-now-cortana-iphone-android/

Philips Hue. (2019, November 12). Smart lighting explained. Philips Hue. https://www2.meethue.com/enus/blog/what-is-smart-lighting

Philips Hue. (2020a). Hue white starter kit E26. Philips Hue. https://www2.meethue.com/en-us/p/hue-whitestarter-kit-e26/046677530365 
Philips Hue. (2020b). Hue white 1-pack E26. Philips Hue. https://www2.meethue.com/en-us/p/hue-white-1-packe26/046677555689

Philips Hue. (2020c). Hue white ambiance starter kit E26. Philips Hue. https://www2.meethue.com/en-us/p/huewhite-ambiance-starter-kit-e26/046677530297

Philips Hue. (2020d). Hue white and color ambiance starter kit E26. Philips Hue. https://www2.meethue.com/enus/p/hue-white-and-color-ambiance-starter-kit-e26/046677548544

Philips Hue. (2020e). Hue dimmer switch. Philips Hue https://www2.meethue.com/en-us/p/hue-dimmerswitch/046677473372

Ring. (2020a). About. Ring. https://shop.ring.com/pages/about

Ring. (2020b). Using Alexa to control your Ring Doorbell. Ring. https://support.ring.com/hc/enus/articles/360031246432-Using-Alexa-to-Control-Your-Ring-Doorbell-

Ring. (2020c). Video doorbell. Ring. https://shop.ring.com/products/video-doorbell

Ring. (2020d). Video doorbell 2. Ring. https://shop.ring.com/products/video-doorbell-2

Ring. (2020e). Video doorbell pro. Ring. https://shop.ring.com/products/video-doorbell-pro

Ring. (2019f). Video doorbell elite. Ring. https://shop.ring.com/products/video-doorbell-elite

Ring. (2020g). Setting up your Ring Doorbell in the Ring App. Ring. https://support.ring.com/hc/enus/articles/115001773266-Setting-Up-Your-Ring-Video-Doorbell-In-the-Ring-App

Ring. (2020h). How to physically install your Ring Video Doorbell with an existing doorbell. Ring. https://support.ring.com/hc/en-us/articles/115001912086-How-to-Physically-Install-Your-Ring-VideoDoorbell-with-an-Existing-Doorbell

Ring. (2020i). How to physically install your Ring Video Doorbell without an existing doorbell. Ring. https://support.ring.com/hc/en-us/articles/115002091706

Ring. (2020j). FAQ. Ring. https://shop.ring.com/pages/faq

Ring. (2020k). Ring Chime. Ring. https://shop.ring.com/products/chime

Watters, A., \& House, A. (n.d.). What is the Ring Doorbell? Dummies: A Wiley Brand. https://www.dummies.com/home-garden/home-security/what-is-the-ring-doorbell/

Welinder, Y. (2019, October 28). Will your smart home product achieve mass adoption? Forbes. https://www.forbes.com/sites/forbesbusinesscouncil/2019/10/28/will-your-smart-home-product-achievemass-adoption/\#57a0a8485f7d

Wilson, C., Hargreaves, T., \& Hauxwell-Baldwin, R. (2014, September 4). Smart homes and their users: A systematic analysis and key challenges. Personal and Ubiquitous Computing, 19(2), 463-476. https://doi.org/10.1007/s00779-014-0813-0

Wilson, C., Hargreaves, T., \& Hauxwell-Baldwin, R. (2017, April). Benefits and risks of smart home technologies. Energy Policy, 103, 72-83. https://doi.org/10.1016/j.enpol.2016.12.047 


\section{Issues in Information Systems}

Volume 21, Issue 2, pp. 43-48, 2020

Wojciechowski, M. (2019, November). New technology: Keeping it ethical, keeping it legal. PT in Motion, 11(10), 30-39. 